\title{
Analogía entre dos estrategias de enseñanza en los dos últimos años
}

\author{
Analogy between two teaching strategies in the last two years
}

\author{
Peñalva María Anahí | Tosti Sonia Beatriz Cecho Analía Cristina \\ https://orcid.org/0000-0001-5835-9110 soniabtosti@yahoo.com https://orcid.org/0000-0001-8210-3633 \\ anahipenalva@gmail.com \\ Facultad de Odontología | Universidad Nacional La Plata | Argentina
}

\section{RESUMEN}

El objetivo de este artículo es el de comparar el rendimiento académico utilizando la dinámica de trabajo en grupos (corrillos) y clase expositiva durante los años 2017 y 2018. El docente formuló preguntas sobre un tema, a diferentes grupos. Luego se constituyeron equipos, conformados por integrantes de distintos grupos. Cada estudiante explicó el tema. Se tomó una evaluación: con corrillos, en el año 2017 donde el $45 \%$ no respondió correctamente ninguna, el $33 \% \%$ respondió 1 , el $20 \%$ contestó 2 , el $2 \% 3$ preguntas y nadie 4 preguntas. En 2018 , con corrillos el $30 \%$ no respondió ninguna pregunta; respondió 1 el $23 \%$, contestó 2 el $28 \%, 3$ el $16 \%$ y nadie respondió las preguntas. En otra aula se expuso con metodología expositiva las 4 preguntas fueron respondidas por el $3 \%$ de los estudiantes. Con clase expositiva en el 2017 el $39 \%$ no contestó correctamente ninguna pregunta, el $66 \% 1$, el $13 \% 2$, el $3 \% 3$ preguntas y ninguno contestó las 4 preguntas. En el año 2018 el $27 \%$ no contestó ninguna, el 31 $\%$ respondió 1 , el $25 \%$ respondió $2,12 \%$ respondió 3 y $4 \%$ respondió correctamente las 4 preguntas. Se observa una diferencia significativa con corrillos entre ambos años.

\section{ABSTRACT}

The objective of this article is to compare academic performance using the dynamics of work in groups and exhibition class during the years 2017 and 2018. The teacher asked questions on a topic, to different groups. Then teams were formed, made up of members of different groups. Each student explained the topic. An evaluation was taken: in groups, in 2017 where $45 \%$ did not answer any correctly, 33\% answered $1,20 \%$ answered 2, 2\% 3 questions and nobody 4 questions. In 2018, in groups 30\% did not answer any questions; 1 answered 23\%, 2 answered 28\%, 3 16\% and nobody answered the questions. In another classroom, the 4 questions were presented with

PALABRAS CLAVE

Equipos,

Corrillos,

Grupos
KEY WORDS

Teams,

Corrillos,

Groups expository methodology and were answered by $3 \%$ of the students. With an exhibition class in 2017, 39\% did not answer any questions correctly, 66\% 1, 13\% 2, 3\% 3 questions and none answered the 4 questions. In 2018, 27\% did not answer any, 31\% answered $1,25 \%$ answered $2,12 \%$ answered 3 and $4 \%$ correctly answered the 4 questions. There is a significant difference with corrillos between both years. 


\section{INTRODUCCIÓN}

La metodología participativa se fundamenta en procesos de intercambio, resolución de problemas y construcción del conocimiento colectivo a través de un conjunto de procedimientos, técnicas y herramientas que implican activamente al alumno en el proceso de enseñanza-aprendizaje En la metodología participativa el eje de la acción educativa está puesto en la persona. Por esa razón creemos que es necesario repensar las estrategias educativas en la Universidad y procurar una enseñanza grupal, centrada en la acción de los estudiantes. Nos parece imprescindible utilizar instrumentos y técnicas que faciliten la participación de los estudiantes y permitan la construcción colectiva de conocimientos.

Las técnicas que favorecen más la actividad de los estudiantes son el interrogatorio, la argumentación, los trabajos en grupo, el estudio dirigido, los debates y las discusiones, la técnica de resolución de problemas, etc. La metodología participativa configura indudables ventajas para el docente. López Noguero (2007 p 108- 109) explicita:

- $\quad$ Ayuda al grupo a reenfocar el tema.

- Fomenta o al menos propicia la creatividad.

- Responsabiliza e involucra al alumno en su propio aprendizaje y en los ritmos de clase.

- $\quad$ Favorece la interrelación personal de los alumnos.

- Puede servirnos para sondear los preconceptos de nuestros alumnos.

- Nos permite cambiar el ritmo de la clase.

- Refuerza a los estudiantes y los motiva.

- Fomenta y ayuda a la reflexión de los miembros de la clase.

- Mantiene la tensión en clase.

- Favorece la atención, la memoria y la agilidad mental.

- Puede suponer un "bang" que constituye una base inicial que favorezca el aprendizaje.

- Desarrolla la capacidad de síntesis, facilita el estudio posterior y favorece la adquisición de conocimientos.

La palabra grupo se incorpora a los usos modernos a través del ita- 
liano groppo (en la forma arcaica) o grupo (más usual hoy) referido a una pintura o una escultura que representa a varios individuos. Desde allí se desliza al lenguaje corriente, en distintas lenguas, para adoptar el significado fundamental de "conjunto de personas". Urbano y Yuni (2015 p 16) explican: "en el uso corriente de nuestro idioma, la palabra grupo refiere al establecimiento de relaciones de asociación, derivadas éstas de la presencia de alguna propiedad común entre las unidades".

Sin embargo, Barreiro (1992 p 13) señala:

"el término grupo (...) es vago. Si bien hay conglomerados humanos que, claramente, no son un grupo como por ejemplo el conjunto de desconocidos que esperan en la fila de un banco sin tener ningún tipo de intercambio o comunicación y otros agrupamientos que con toda certidumbre constituyen un grupo (mi pequeño grupo de amigos de la adolescencia); hay una zona de penumbra donde aparecen situaciones que no sabríamos como encuadrar (...) es importante establecer una diferencia entre lo que podríamos llamar grupo en sentido estricto y ciertos conjuntos o agrupamientos de personas que aunque se hallan juntas físicamente, y en un número suficientemente pequeño para constituir un grupo, no interactúan o lo hacen sólo de manera circunstancial".

Entonces, se considera un grupo a un conjunto de personas que poseen una interacción estratégica mutua y de conjunto frecuente, un objetivo compartido, una noción de pertenencia y una cierta interdependencia funcional, de modo que las actitudes o reacciones de un integrante incide en las de los otros. Su naturaleza es compleja y articula dimensiones polares como lo objetivo / lo subjetivo; lo social/ lo psicológico; lo material / lo simbólico; lo explícito / lo implícito; lo manifiesto / lo latente; etc. Se produce un cierto modelo de relación interpersonal con un sistema de valores más o menos implícito, con una distribución de funciones para los distintos miembros, que se hallan en una situación dinámica porque su existencia se apoya en la presencia de ciertas propiedades que alcanzan un equilibrio dinámico. A su vez conforma un espacio social que se integra a organizaciones e instituciones insertándose en la estructura social. Así se constituye un nuevo sujeto social, que es definido por el nosotros.

Los grupos se constituyen a través de un proceso a través del cual se consolidan ciertas pautas de conducta, ciertos códigos con respecto a su accionar, se cristalizan algunos vínculos, alianzas, atracciones o rechazos, afectos intensos entre algunos y marginación de otros. Mientras circula el conocimiento, se va desarrollando una trama vincular, que puede desembocar en una mayor o menor participación de un integrante que otro, una mayor o menor confianza o descalificación. 
Las técnicas grupales constituyen la concreción de una serie de objetivos pedagógicos en los que el grupo se considera el eslabón básico para que el estudiante adquiera, elabore e integre conocimientos, habilidades o actitudes.

\section{Las técnicas grupales constituyen la concreción de una serie de objetivos pedagógicos en los que el grupo se considera el eslabón básico para que el estudiante adquiera, elabore e integre conocimientos, habilidades 0 actitudes.}

García, citado por López Noguero (2007 p 108), afirma que "se trata de maneras, procedimientos, o medios sistematizados de organizar y desarrollar la actividad educativa del grupo, donde la propia experiencia y conocimiento de los participantes se convierte en el más importante de los materiales con los que hay que trabajar". A la vez, Fuentes y otros, citados por el mismo autor (2007, p 108) sostienen que "las técnicas grupales tienen, sobre todo, una doble finalidad: método válido para la transmisión comprensiva y objeto de ratificación de esos conocimientos aprendidos y "aprehendidos".

Entre las técnicas de dinámica grupal sin expertos encontramos la comisión, el seminario, el equipo de trabajo, el estudio de casos, el diálogo, el foro y el Philips 66. Esta última técnica fue denominada en el año 1964 como el Método de reunión en corrillos por Beal, Bohlen y Neil Raudabaugh en su libro "Conducción y acción dinámica del grupo", por lo que se considera que la actual técnica de corrillos, que también es una técnica sin expertos, deriva del Philips 66.

Este artificio de descomponer un grupo grande en unidades pequeñas a fin de facilitar la discusión fue descrito y divulgado por J. Donald Philips, de la Universidad del Estado de Michigan (Michigan State College). En esencia consiste en dividir cualquier grupo en otros más pequeños; de allí que los corrillos deriven de ella. En el libro Metodología participativa en la Enseñanza Universitaria (2007 p 166 y p 169) Fernando López Noguero las describe como dos técnicas diferentes.

Características dinámicas que se aplican tanto al Philips 66 como a los corrillos (Basado en Beal, Bohlen y Neil Raudabaugh 1964, p.172- 173):

1. Permite y propicia la creación de una atmósfera informal y de respetuoso compromiso, por grande que sea el grupo.

2. Permite la participación de todos los presentes.

3. Estimula la reunión de las ideas dentro del grupo pequeño y por medio de la información dada al grupo mayor, se facilita la comunicación y participación de todos.

4. Alienta la división del trabajo y de la responsabilidad.

5. Asegura la máxima identificación individual total con el problema que se trata.

6. Es una técnica rápida para obtener un acuerdo. 
7. Estimula el desarrollo de la confianza individual con el proceso democrático.

8. Torna muy difícil el control de la discusión y de las recomendaciones por conductores autoritarios o por pequeñas minorías muy expansivas.

9. Ayuda a liberar a los individuos de sus inhibiciones para participar mediante la identificación de sus ideas con un grupo pequeño que puede después llegar a ser el protagonista principal.

10. La técnica a menudo alivia la fatiga, el aburrimiento y la monotonía que se observan cuando las reuniones grandes tienden a estancarse.

Ambas técnicas pueden elegirse para lograr que el grupo informe en cuanto a sus intereses, necesidades, problemas, deseos y sugerencias, que luego se emplearán en el planeamiento de los programas, de las actividades, de los procedimientos de evaluación y de las normas de conducta. También pueden elegirse para despertar el interés del auditorio al comenzar un programa. El interés, la preocupación y la identificación del grupo puede suscitarse muy rápidamente por esta técnica y se puede establecer una posición mental para escuchar y aprender. Con estas técnicas, a menudo, se llega a sugerencias útiles para la acción o para lograr soluciones finales.

Estas técnicas son útiles cuando hay un deseo de ampliar la base de comunicación y de la participación, cuando se desea recurrir a la totalidad de los recursos de los integrantes del grupo en relación con el problema de que se trata, cuando se necesita analizar un problema complicado que puede ser separado lógicamente en partes componentes y asignarse dichas partes a diversos grupos de reunión en corrillos, cuando parece deseable ampliar la responsabilidad asegurándose de la participación de todos los integrantes. Puede extender su alcance asignando secciones separadas a grupos diversos y atribuyendo papeles específicos a varios individuos dentro de cada grupo. Asimismo es de utilidad cuando parece importante crear una atmósfera informal y democrática, cuando se necesita una rápida reunión de ideas en un grupo grande, cuando se desea obtener un acuerdo, cuando se aspira a crear la identidad del individuo con el grupo o su problema, cuando se quiere intentar desarrollar la seguridad y la confianza individual en la solución de problemas. También es útil cuando se desea estimular la motivación mediante un cambio de técnicas, cuando las normas y los controles sociales son inadecuados en el grupo mayor, ya que la reunión en corrillos es una situación que "obliga" a pensar y discutir. Por último el Philips 66 y los corrillos son útiles cuando uno desea sacar el máximo de provecho de la heterogeneidad del grupo: los que se encuentran en diversos niveles de experiencia, de conocimiento o de antecedentes, están mucho más dispuestos a hablar en el grupo pequeño y a expresar las ideas de la minoría y cuando el asunto es de tal naturaleza que se puede discutir con más facilidad en un grupo pequeño.

Para emplear estas técnicas, 1) el grupo deberá tener claros los objetivos de la reunión y la sección de la reunión en que esta técnica 
sea apropiada, 2) considerar cualquier medio alternativo que pueda producir resultados igualmente buenos, 3) pensar a fondo para que propósitos puede emplearse esta técnica y 4) tener preparadas con anticipación las preguntas que se han de hacer a los grupos de reunión en corrillos; si fuere necesario, escritas en tarjetas.

La técnica de corrillos consiste en dividir un grupo de personas en varios subgrupos con el propósito fundamental de analizar y discutir determinada información, para obtener conclusiones. Sus objetivos son: obtener una visión general de preconceptos de los estudiantes, fomentar la participación activa de los mismos, favorecer el diálogo y la integración y responsabilizar al estudiante de su propio aprendizaje. Cirigliano y Villaverde (1986 p148) proponen como objetivos de esta técnica, además de promover la participación activa de todos los miembros de un grupo y obtener sus opiniones en un tiempo breve, llegar a la toma de decisiones, obtener puntos de vista acerca de un problema o cuestión, desarrollar la capacidad de concentración y de síntesis del pensamiento de cada pequeño grupo, para que sea difundida en beneficio de todos. El objetivo principal, según estos autores, consiste en lograr una participación democrática en los grupos numerosos.

Su tiempo de duración depende del tema tratado, por lo general es de 30 a 50 minutos. Al comienzo, el profesor explica a todos los participantes:

- Los propósitos que se persiguen y los resultados que se pretenden alcanzar.

- La participación que se espera de cada uno.

- El tiempo que se dispone para presentar los resultados.

- Descripción de la manera como se forman los corrillos.

- Distribución del mobiliario del aula para trabajar.

- Aclarar dudas antes de seguir.

- Invitar a realizar los corrillos y pedir a cada corrillo nombrar un moderador y un portavoz.

- El moderador deberá conducir el análisis y la discusión, controlar dificultades y evitar desviaciones.

- El portavoz llevará un registro de actividades y deberá escribir y dar a conocer las conclusiones.

Se forman equipos de dos a seis personas, dependiendo del número total de integrantes del grupo total. El profesor debe procurar que los grupos formados no sean los mismos siempre. Si es así, podrá intervenir él en la formación de los grupos.

Una vez divididos en grupos, el docente formula distintas preguntas sobre un tema, las cuales se repartirán en tarjetas a cada equipo. Luego los grupos debaten y realizan intercambios verbales para responder a las diversas preguntas formuladas. A continuación, los estudiantes se 
agrupan por segunda vez, pero cada equipo se hallará conformado por integrantes de los distintos grupos producto de la división anterior. El moderador explica a sus compañeros del nuevo grupo el tema sobre el que trabajó. El portavoz de cada equipo arriba a las conclusiones y las da a conocer a toda la clase.

Objetivos: Comparar el rendimiento académico entre estudiantes que tuvieron una experiencia con metodología participativa de corrillos y estudiantes que recibieron una clase expositiva tradicional.

Metodología: Se trabajó con dos grupos A y B. En el grupo A tuvo lugar la clase con metodología participativa, se realizó una primera división en cuatro equipos de cinco estudiantes cada uno. Una vez divididos, el docente formuló distintas preguntas sobre un tema, a cada equipo. Luego los grupos debatieron y realizaron intercambios verbales para responder a las diversas preguntas formuladas. Mientras, el docente observó el desarrollo de la actividad y resolvió las dudas planteadas por los estudiantes. A continuación, los estudiantes se agruparon por segunda vez, pero cada grupo se hallaba conformado por integrantes de los distintos grupos producto de la división anterior. Cada estudiante explicó a sus compañeros del nuevo grupo el tema sobre el que trabajó. Se tomó una evaluación que constó de cuatro preguntas abiertas. En el grupo $B$ se dio una clase expositiva tradicional.

Resultados: Con corrillos, en el año $2017,45 \%$ no respondió correctamente ninguna pregunta, el $33 \%$ \% respondió 1; el 20\% contestó 2; el $2 \%$ 3 preguntas y nadie 4 preguntas. En 2018, con corrillos el $30 \%$ no respondió ninguna pregunta, respondió 1 el $23 \%$, contestó 2 el $28 \%$; 3 el $16 \%$ y nadie respondió las preguntas. En otra aula expusieron con metodología expositiva las 4 preguntas el $3 \%$. Con clase expositiva, en el 2017, el $39 \%$ no contestó correctamente ninguna pregunta, el $66 \% 1$; el $13 \% 2$; el $3 \%$ 3 preguntas y ninguno contestó las 4 preguntas. En el año 2018, el $27 \%$ no contestó ninguna; el $31 \%$ respondió 1; el 25\%respondió 2; el 12\% respondió 3 y $4 \%$ respondió correctamente las 4 preguntas. Se observa una diferencia significativa con corrillos entre ambos años.

Discusión: La importancia de la participación de los estudiantes en el aprendizaje de los contenidos y la concepción del aprendizaje como construcción, ha orientado las investigaciones didácticas, los planteos teóricos y los modelos de intervención en el aula en los últimos años. Frente a la concepción tradicional, con la cual el estudiante era un mero receptor de conocimientos, en la concepción constructivista el estudiante es sujeto de su propio aprendizaje, con un rol activo en que se pone énfasis en las estrategias dinámicas de aprendizaje, que promueven los procesos inferenciales, la creatividad y la investigación.

\section{La importancia de la participación de los estudiantes en el aprendizaje de los contenidos y la concepción del aprendiza- je como construcción, ha orientado las investigaciones didác- ticas, los planteos teóricos y los modelos de intervención en el aula en los últimos años.}


Desde nuestra práctica consideramos que es necesario procurar una enseñanza grupal, centrada en la acción de los estudiantes. Nos parece imprescindible utilizar instrumentos y técnicas que faciliten la participación de los estudiantes y permitan la construcción colectiva de conocimientos. De esta manera, se intenta plasmar en el aula técnicas grupales que permitan el tratamiento de un tema o la formulación de un concepto clave, a través de la participación del estudiantado.

Se considera que la actual técnica de corrillos deriva del Philips 66. Esta técnica grupal fue descrita y divulgada por J. Donald Philips, de la Universidad del Estado de Michigan. Ambas técnicas tienen en común el hecho de descomponer un grupo grande en unidades pequeñas a fin de facilitar la discusión. Actualmente hay poca literatura y trabajos de investigación que den cuenta de su aplicación.

Conclusiones: Se observa una diferencia significativa con corrillos entre los tres años y con respecto a los que no contestaron ninguna pregunta. Solamente en el año 2015 hubo estudiantes que respondieron correctamente las cuatro preguntas tanto en los que trabajaron con corrillos como en los que recibieron clases expositivas. Esta innovación de las prácticas docentes debe entenderse como una mejora colaborativa que genere ilusión y compromiso, que estimule nuevos aprendizajes y forme a los profesores como agentes responsables de desarrollar reformas en la práctica. 


\section{BIBLIOGRAFIA}

Barreiro T. (1992). "Trabajos en grupo Buenos Aires". Buenos Aires: Kapeluz

Beal G., Bohlen J. y Neil Raudabaugh J. (1964). "Conducción y acción dinámica del grupo-Biblioteca de Psicología contemporánea". Buenos Aires: Kapeluz.

Cirigliano, G. F.; Villaverde, A. (1986). “Dinámica de grupos y educación". Buenos Aires: Humanitas.

López Noguero, F. (2007). “Metodología participativa en la Enseñanza Universitaria". Madrid: Narcea.

Perrone, G. y Propper, F. (2007). “Diccionario de Educación”. Buenos Aires: Alfagrama.

Urbano, C. y Yunl, J. (2015). "El trabajo grupal en las instituciones educativas". Córdoba: Brujas. 